$54^{\text {ème }}$ Congrès de la SFMBCB, 03018 (2011)

DOI: $10.1051 / \mathrm{sfmbcb} / 20115403018$

(C) Owned by the authors, published by EDP Sciences, 2011

\title{
Influence d'une bactérie sulfato-réductrice (Desulfovibrio fairfieldensis) sur le comportement en corrosion du titane
}

\author{
Sourdot A, Le A, Bally J, Curien R, Bravetti P \\ UFR d'Odontologie, Département de Chirurgie buccale, Nancy, France \\ alex.sourdot@voila.fr
}

Contexte: Depuis de nombreuses années, il a été montré dans l'industrie que la corrosion bactérienne, en particulier celle due aux bactéries sulfato-réductrices, pouvait causer des dommages importants sur les métaux immergés dans un milieu aqueux. Malgré une résistance à la corrosion élevée, le titane est soumis à l'agressivité du milieu buccal et son film d'oxyde protecteur peut être altéré dans certaines conditions déjà bien documentées (Strietzel 1998, Schiff et al. 2002). Cependant, très peu d'études relatent les effets des micro-organismes de la flore buccale sur la résistance à la corrosion des alliages dentaires (Laurent et al. 2001, Chang et al. 2003)

Objectif: Cette étude présente l'élaboration d'un protocole permettant la mise en présence de bactéries sulfato-réductrices appartenant à l'espèce Desulfovibrio fairfieldensis (Df) et de titane. Elle montre l'influence du Df sur le comportement en corrosion du titane des implants en fonction de son état de surface (lisse ou rugueux). Le choix de la bactérie a été guidé par des études récentes (Langendijk et al. 2001, Loubinoux et al. 2002) qui ont mis cette espèce en évidence dans les poches parodontales.

Méthode : Avant tout essai, la surface et les coupes des implants ont été observées en microscopie électronique à balayage, et la sonde de Castaing a été utilisée pour analyser la composition atomique exacte des implants étudiés. Après caractérisation de la croissance bactérienne en bouillon Wilkins Chalgren enrichi, des tests d'adhésion ont été réalisés. L'adhésion des bactéries aux surfaces implantaires a été examinée en microscopie électronique. Enfin des paramètres électrochimiques ont été mesurés lors des essais de mise en présence titane/ bactéries.

Résultats : L'adhésion du Df aux surfaces implantaires lisses a été confirmée. Le Df semble adhérer préférentiellement sur les parties lisses.

Les variations des mesures de potentiel et de corrosion (Ecorr), les courants de corrosion (Icorr) et le palier de passivation (Ipass) tendent à montrer une diminution de la résistance à la corrosion du titane en présence du Df. L'état de surface (lisse ou rugueux) n'a pas influencé la cinétique de corrosion.

Conclusion : La présence du Df semble conduire à une augmentation de la cinétique de corrosion du titane. L'existence d'un biofilm reste à démontrer. Cette étude est un pré-requis pour mieux caractériser le comportement en corrosion du titane en présence du Df sur une période plus longue.

This is an Open Access article distributed under the terms of the Creative Commons Attribution-Noncommercial License 3.0, which permits unrestricted use, distribution, and reproduction in any noncommercial medium, provided the original work is properly cited. 